\title{
An electrochemical biosensor for rapid detection of anti-dsDNA antibodies in absolute scale.
}

\author{
Pablo Fagúndez ${ }^{1}$, Gustavo Brañas ${ }^{1}$, Justo Laíz ${ }^{1}$, Juan Pablo Tosar $^{1,2^{*}}$ \\ ${ }^{1}$ Nuclear Research Center, Faculty of Science. Universidad de la República. Montevideo 11400. \\ Uruguay. ${ }^{2}$ Functional Genomics Unit. Institut Pasteur de Montevideo. Montevideo 11400. \\ Uruguay. *Email: jptosar@cin.edu.uy
}

Keywords: Autoantibodies, Lupus, sensor, anti-dsDNA, point-of-care

\section{Abstract}

Autoimmune diseases are chronic inflammatory pathologies that are characterized by the presence of antibodies against own epitopes in serum (autoantibodies). Systemic lupus erythematosus (SLE) is a common autoimmune pathology, characterized by the presence of antinuclear antibodies (ANAs). These include anti-dsDNA (a-dsDNA) antibodies, which are widely used for diagnosis and disease monitoring. Their determination is carried out by traditional techniques such as Indirect Immunofluorescence (IFI) or Enzyme Linked Immunosorbent Assay (ELISA), which are time consuming, require qualified technicians, and are not compatible with decentralized analysis outside a laboratory facility. Here, we show a sandwich-format electrochemical biosensor-based method for $\alpha$-dsDNA determination in a rapid and simple manner. Total assay time is only 30 minutes and the sensor is capable of detecting $16 \mathrm{ng}(8 \mu \mathrm{g} / \mathrm{mL})$ of $\alpha$-dsDNA antibodies. Using the current derived from the detection limit of the method as a cut-off, we could discriminate positive from negative serum samples with $90 \%$ sensitivity and 100\% specificity. By using monoclonal antibodies for calibration curves, our results are presented in absolute scale (i.e., concentration instead of serum title) what will help to perform comparisons between methods and further improvements of this protocol. In an effort to render the sensor compatible with automation, we minimized manipulation steps without compromise of the analytical performance, even in complex samples such as serum. 


\section{Introduction}

2 Autoimmune disorders are chronic inflammatory pathologies that affect over 5-8\% of the world

3 population ${ }^{1}$. In these disorders, the immune response of the individual is directed against its

4 own components causing tissue- or organ-specific damage, generating local or systemic

5 responses. Common autoimmune diseases include systemic lupus erythematosus (SLE),

6 rheumatoid arthritis, multiple sclerosis, systemic sclerosis, type 1 diabetes, inflammatory bowel

7 disease, and antiphospholipid syndrome. Diagnosis of these diseases is challenging for

8 professionals, since their symptoms can vary between individuals and overlap with other rare

9 pathologies ${ }^{2,3}$. Moreover, their origin is still now difficult to understand because of the genetic

10 and environmental factors involved in their appearance ${ }^{4}$. However, they share some common

11 features, such as the presence of antibodies directed against own epitopes (autoantibodies).

12 SLE, considered as a model of autoimmune diseases, has been extremely studied and it has been

13 reported more than a hundred autoantibodies which involve reactivity against nuclear,

14 cytoplasmic and membrane components. The antinuclear antibodies (ANAs) are reactive against

15 single and double strand DNA, histones, nucleosomes and chromatin, as well as other nuclear

16 antigens (Ro, La and Sm ribonucleoproteins). ${ }^{5}$ Specific antibodies are associated with distinct

17 clinical features. For example, the anti-dsDNA antibodies ( $\alpha$-dsDNA) are associated with the

18 development of Lupus nephritis. Total ANAs, $\alpha$-dsDNA and $\alpha$-Sm antibodies are considered as

19 the hallmarks of this pathology, and are included in the serological American College of

20 Reumathology (ACR) criteria for diagnosis. ${ }^{6-10}$ SLE is also characterized by flares and

21 remissions steps ${ }^{8,11}$. Thus, the determination of the aforementioned antibodies in serum samples

22 is relevant not only for diagnosis but also for classification, determination of the state of the

23 disease, as well as for therapeutic evaluation and drug adjustment. ${ }^{3,12}$

24 The gold standard technique for ANA determination is Crithidia luciliae indirect

25 immunofluorescence (CLIF). In this assay, fluorescence intensity is used for title determination,

26 and, in addition, it can be defined the specific autoantibodies contained in the sample because of 
27 the different staining patterns. Although several commercial kits have been developed with

28 different cellular substrates, these require complex instrumentation and experienced stuff, being

29 unsuitable for point-of-care applications. ${ }^{13-15}$ Recently, Enzyme Linked Immunosorbent Assay

30 (ELISA) kits have been developed and commercialized. These kits use single or multiple antigen-coated wells (purified or synthetic), such as Ro, La, and dsDNA. However, diversity in antigen and adsorption strategies can affect comparability of the results between labs using kits from different suppliers. Line immuno semi-quantitative assays (variation of immuno-blots) are easy to use and facilitate detection of multiple autoantibodies in one strip. Nevertheless, they generally offer low sensitivity and specificity for certain antibodies. ${ }^{16}$

All the assays described above are time-consuming or can only be developed in a centralized laboratory with certificated equipment and technicians, being unable to provide immediate results for flare prediction and drug adjustment therapy. Biosensors emerge as suitable platforms for quick point-of-care tests. A biosensor can be described as an analytical device that includes a biological component as sensor, which works in association to a physicochemical transducer. ${ }^{17,18}$ Most biosensors oriented to the detection of autoantibodies employ a dsDNAcoated surface (or other auto-antigen) which is used to capture the autoantibodies in the sample.

43 These can be detected directly (label-free) or indirectly (e.g., with the help of an enzymecoupled secondary antibody). Some label-free approaches include Surface Plasmon Resonance chips (SPR) or Quartz Crystal Microbalance (QCM) measurements. ${ }^{13,19-21}$ However, electrochemical label-based biosensors are very promising for point-of-care tests because of the

47 low costs associated with their automatization and miniaturization. ${ }^{22}$ In this regard, 48 Konstantinov, Rubin and coworkers have developed an electrochemical sandwich-type immunoassay where nuclear antibodies are detected through current measurements (using redox enzyme-coupled secondary antibodies). ${ }^{23-25}$

51 Recently reported electrochemical biosensors as well as commercial kits use a WHO52 standardized serum as a reference to measure autoantibody concentrations. Thus, concentrations 53 are reported either in arbitrary units or in title units (maximum serum dilution to obtain a signal 
54 with a suitable signal-to-noise ratio), which are compared against the WHO reference or other

55 previously calibrated positive samples. This is suitable for most clinical applications, but we

56 argue that results expressed in absolute scale (i.e., molarity or grams per liter) are convenient for

57 both basic research and applications where the inclusion of a previously calibrated control is not

58 always possible (e.g., self-monitoring). Furthermore, the WHO-standardized reference serum is

59 not available any longer. ${ }^{2}$

60 The previous reasons pushed us to develop an amperometric biosensor capable of detecting $\alpha$ -

61 dsDNA antibodies, which could serve as a diagnostic and disease tracing tool in the near future.

62 We also propose a methodology to obtain absolute $\alpha$-dsDNA antibody concentrations (in terms

63 of mass per volume), without the need of positive and previously calibrated human sera.

64 Materials and methods

\section{$65 \quad$ Reagents and solutions}

Lyophilized genomic salmon sperm DNA (“dsDNA”, Sigma-Aldrich, Cat.No. D1626) was employed for ELISA assays and electrode modification. Poly-L-Lysine and 3,3',5,5' tetramethylbenzidine (TMB, $\geq 98 \%$ ) were purchased from Sigma-Aldrich. Freeze-dried bovine serum albumin (BSA $\geq 96 \%$ ) was obtained from Spectrum Chemical Mfg Corp. (USA) and $1 \%$ fresh solutions were made in phosphate buffer saline ("PBS": 10mM sodium phosphate buffer, $150 \mathrm{mM} \mathrm{NaCl}, \mathrm{pH}=7.4)$. Mouse monoclonal anti-dsDNA antibodies ( $\alpha$-dsDNA: sc-58749), normal mouse immunoglobulins (m IgG: sc-2025) and mouse monoclonal anti-human CD9 antibodies ( $\alpha$-CD9: sc-13118) were purchased from Santa Cruz Biotechnology INC. Rabbit HRP-conjugated anti-mouse IgG ( $\alpha$-m-IgG/HRP, ab6728) was purchased from Abcam. Fetal bovine serum (FBS) was supplied by Gibco. Stock DNA concentrations were estimated using a

77 NanoDrop 1000 spectrophotometer (Thermo Scientific). Screen-printed three or four (bi-

78 electrode) electrode strips (work: carbon; counter: carbon, pseudoreference: silver) were 
79

80

\section{ELISA assay}

82 The ELISA assay was carried out as previously described. ${ }^{26}$ A 96-well ELISA plate 83 (CELLSTAR, Cat.-No.655 180) was pretreated with $100 \mu \mathrm{L}$ of $50 \mu \mathrm{g} / \mathrm{mL}$ poly-L-Lysine

supplied by DropSense (Oviedo, Spain). All electrochemical measurements were carried out with a CHI760D workstation (CH Instruments).

solution (in water) for $30 \mathrm{~min}$ at room temperature (RT) and washed three times with Tris Borate Saline buffer ("TBS", $10 \mathrm{mM}, 150 \mathrm{mM} \mathrm{NaCl} \mathrm{pH}=7.5$ ). Then, $100 \mu \mathrm{L}$ of dsDNA solution ( $4 \mu \mathrm{g} / \mathrm{mL}$ in TBS) was incubated for $60 \mathrm{~min}$ at $\mathrm{RT}$ and washed in the same way as in the previous step. After this, the plate was blocked over-night with $100 \mu \mathrm{L}$ of $1 \%$ BSA solution at RT, and washed vigorously three times with PBS- $0.1 \%$ Tween-20 buffer. Then, $100 \mu \mathrm{L}$ of PBS dilution containing either $\alpha$-dsDNA or $\mathrm{m}$ IgG (specific and unspecific antibodies respectively) was incubated for $60 \mathrm{~min}$ at $37^{\circ} \mathrm{C}$ and washed with $\mathrm{PBS}-0.1 \%$ Tween as previously described. $100 \mu \mathrm{L}$ of the $\alpha$-m-IgG/HRP (1/2000 dilution, plus $1 \%$ BSA) was dispensed and incubated for $60 \mathrm{~min}$ at $37^{\circ} \mathrm{C}$. The plate was washed and finally incubated for 30 min with $100 \mu \mathrm{L}$ of TMB- $\mathrm{H}_{2} \mathrm{O}_{2}$ solution $\left(2 \mathrm{mM}\right.$ TMB, $1 \mathrm{mM} \mathrm{H}_{2} \mathrm{O}_{2}$, diluted in $50 \mathrm{mM}$ acetic acid/sodium acetate buffer, $\mathrm{pH}=5$ ). The reaction was stopped with $50 \mu \mathrm{L}$ of $5 \mathrm{M} \mathrm{HCl}$ and the absorbance at $450 \mathrm{~nm}$ was measured in a microplate reader (Thermo Scientific, Multiskan EX).

\section{Construction of dsDNA-modified electrodes}

$100 \mathrm{uL}$ of acetic acid/sodium acetate buffer $(200 \mathrm{mM}, \mathrm{pH}=5)$ was placed in order to cover the three or four screen-printed electrodes in each strip, and a potential of $+1.7 \mathrm{~V}$ was applied for $120 \mathrm{~s}$ as electrode pretreatment, followed by three washes with the same buffer. A dsDNA solution $(0.5 \mu \mathrm{g} / \mu \mathrm{L}$ in acetic/acetate buffer) was prepared and vortexed for $30 \mathrm{~s}$ and then $50 \mu \mathrm{L}$ were placed on the electrode system. A constant potential of $+0.5 \mathrm{~V}$ was applied for $300 \mathrm{~s}$ for dsDNA immobilization. After washing three times with PBS, $2 \mu \mathrm{L}$ of $1 \%$ BSA were deposited (on the working electrode only) and incubated for $30 \mathrm{~min}$ at $37^{\circ} \mathrm{C}$ in a wet chamber with $100 \%$ humidity. 
105 The dsDNA immobilization was confirmed by cyclic voltammetry (CV) after successive

106 washing steps. Briefly, $50 \mu \mathrm{L}$ of acetic acid buffer were placed on the three electrode system

107 and $\mathrm{CV}$ was carried out between $-0.2 \mathrm{~V}$ and $+1.0 \mathrm{~V}$ at a scan rate of $0.05 \mathrm{~V} / \mathrm{s}$ in order to observe

108 the guanine oxidation signal.

\section{Detection of specific antibodies}

Different dilutions of $\alpha$-dsDNA and $\mathrm{m} \mathrm{IgG} \mathrm{were} \mathrm{prepared} \mathrm{in} \mathrm{PBS.} \mathrm{Two} \mathrm{microliters} \mathrm{of} \mathrm{these}$

solutions were placed on the working electrode and incubated for $20 \mathrm{~min}$ at $37^{\circ} \mathrm{C}$ in a wet chamber. For the bi-electrode strips, each antibody was placed in a different working electrode.

After this, the electrodes were washed three times with PBS and $2 \mu \mathrm{L}$ of $\alpha$-m-IgG/HRP antibody $(1 / 2000,1 \% \mathrm{BSA})$ was added and incubated under the same experimental conditions.

The electrodes were then washed with PBS and $50 \mu \mathrm{L}$ of a TMB/ $\mathrm{H}_{2} \mathrm{O}_{2}$ solution was added. Immediately, the working electrode was placed at a constant potential of $-0.1 \mathrm{~V}$ and the TMB reduction current was registered. For optic measurements, only monoelectrodes were employed. After antibody incubations, the $\mathrm{TMB} / \mathrm{H}_{2} \mathrm{O}_{2}$ solution was incubated for $5 \mathrm{~min}$, followed by the addition of $5 \mu \mathrm{L}$ of $5 \mathrm{M} \mathrm{HCl}$. Two microliters of the mixture were measured at $450 \mathrm{~nm}$ using a NanoDrop spectrophotometer.

\section{Performance in serum samples}

122 As an approximation to the analysis of real samples, assays were performed in the bioelectrode strips by spiking $\alpha$-dsDNA or irrelevant antibodies directly in 1/80 FBS. Here, two different strategies were tested. One strategy (which we called the "two-step" method) consisted in incubating $\alpha$-dsDNA-containing FBS on the working electrode, performing two wash steps with PBS, and then incubating ( $20 \mathrm{~min}$ at $37^{\circ} \mathrm{C}$ ) the electrodes with $2 \mu \mathrm{L}$ of $\alpha-\mathrm{m}-\mathrm{IgG} / \mathrm{HRP}$ antibody (1/2000 dilution, plus $1 \%$ BSA) followed by a new washing step and addition of the $\mathrm{TMB} / \mathrm{H}_{2} \mathrm{O}_{2}$ solution. In contrast, the "one-step" method consisted in preincubating the $\alpha$-dsDNA (or irrelevant antibodies) with the $\alpha$-m-IgG/HRP in FBS. Briefly, $5 \mu \mathrm{L}$ of $\alpha$-dsDNA (in $1 / 40$ FBS) 
131 the mixture was placed on the working electrode and the procedure continued the same way as

132 described above. As a consequence, one single incubation with the sample and HRP-conjugated

133 antibodies was needed, avoiding the washing step in between, and reducing assay time

134 approximately two-fold.

\section{Results}

136 We propose the construction of an amperometric biosensor capable of detecting anti-double

137 stranded DNA antibodies, which offer diagnostic and prognostic value in several auto-immune

138 diseases. This biosensor is based on the specific binding of $\alpha$-dsDNA antibodies present in a test

139 sample to dsDNA molecules immobilized on the surface of a disposable screen-printed carbon

140 graphite electrode, and the subsequent binding of anti-mouse IgG antibodies conjugated to the

141 electroactive enzyme HRP (conjugated $\mathrm{ABs}$ ). The electrochemical reduction the oxidized TMB

142 generated by the catalysis of HRP is measured ${ }^{27}$, what is related to the amount of $\alpha$-dsDNA

143 antibodies located in the sensor's surface. In an attempt to render this biosensor compatible with

144 automatization, the conjugated $\mathrm{ABs}$ were directly introduced in the test sample, greatly

145 simplifying the detection procedure (one-step method, Scheme 1).

\section{Specificity of the $\alpha$-dsDNA antibody}

147 Specificity of our $\alpha$-dsDNA antibody was studied by ELISA (Figure 1). Wells sensitized with

148 either vortexed or intact dsDNA showed a characteristic binding response when incubated with

149 increasing concentrations of the mouse monoclonal $\alpha$-dsDNA antibody, with a linear response

150 below $0.07 \mu \mathrm{g} / \mathrm{mL}$. In contrast, incubation with normal mouse immunoglobulins (m $\operatorname{IgG})$

151 showed basal absorbance independently of the concentration used, confirming specificity of the

152 assay. Secondly, we assayed the $\alpha$-dsDNA antibody against genomic DNA, plasmid DNA and a

153 purified PCR product, confirming that it is the dsDNA molecule itself (rather than co-purified

154 nucleosomes present in certain genomic DNA preparations) which is being recognized by the

155 antibody used throughout this study. (Supplementary Figure 1). 


\section{Electrode modification}

157 Deposition of dsDNA on the working electrode was done at a constant potential as indicated in

158 Methods. We asked whether that immobilization method would be suitable for maintaining the 159 dsDNA attached to the electrode surface during the whole procedure. To test this, we subjected 160 dsDNA-modified BSA-blocked electrodes to a number of washes in PBS, which was equivalent 161 to the number of washes in our longest assay (three washing cycles, three washes per cycle). 162 The presence of DNA in the electrodes was then analyzed by measuring the irreversible guanine 163 oxidation signal ${ }^{28}$ at $+0.83 \mathrm{~V}$ by cyclic voltammetry, and comparing this signal with naked (Figure 2A) or BSA-only electrodes (data not shown).

\section{Specificity of the electrochemical biosensor}

The electrochemical behavior of TMB on our screen-printed dsDNA-modified carbon electrodes was studied by cyclic voltammetry (Supplementary Figure 2) in order to determine the working potential where electrochemical TMB oxidation was virtually zero, and also registering the electrochemical reduction of oxidized TMB. By doing so, we defined $-0.1 \mathrm{~V}$ as a suitable applied potential for subsequent constant potential assays.

As a proof-of-principle, intensity vs time ( $\mathrm{I}$ vs t) assays at $-0.1 \mathrm{~V}$ are shown in Figure $2 \mathbf{B}$. Incubation of dsDNA-modified electrodes with the $\alpha$-dsDNA monoclonal antibody (followed by detector $\mathrm{ABs}$ and $\mathrm{TMB} / \mathrm{H}_{2} \mathrm{O}_{2}$ solution) showed the expected response ${ }^{27}$, with a negative (i.e., reduction) signal decreasing in absolute value with time, and approaching a concentrationdependent plateau as predicted by the Cottrell equation. ${ }^{29}$ Here, total TMB concentration is constant, but the concentration of oxidized TMB depends on the number of HRP molecules present at the electrode's surface. In contrast, electrodes incubated with normal mouse IgG displayed a negligible signal, comparable to electrodes only exposed to the conjugated ABs. This finding demonstrates that unspecific binding of conjugated $\mathrm{ABs}$ to our electrodes is despicable in the absence of analyte (i.e., $\alpha$-dsDNA antibodies). 


\section{Optical and electrochemical response}

182 Based on the fact that our electrochemical signal depends on the concentration of enzymeoxidized TMB, which also absorbs visible light with a maximum at $450 \mathrm{~nm}$ after acidification of the medium, we compared the analytical performance of the sensor by both optical and electrochemical readouts. Similarly to what was previously observed in ELISA plates (Figure 1), the absorbance of oxidized TMB was saturated above $0.05 \mu \mathrm{g} / \mathrm{mL}$ of $\alpha$-dsDNA antibodies in carbon/dsDNA/BSA electrodes (Figure 3A), with no signs of unspecific binding of antibodies to the electrode surface. In contrast, the electrochemical response (measured as the electrical current in I vs t plots at exactly $\mathrm{t}=100 \mathrm{~s}$ ) was linear up to approximately $0.5 \mu \mathrm{g} / \mathrm{mL}$, showing a 10-fold increase in the dynamic range of the method when compared to absorbance measurements. It is important to mention that this assays where performed in bi-electrodes, where both working electrodes were modified simultaneously using the same dsDNA solution, and incubated with either $\alpha$-dsDNA antibodies or normal mouse IgGs at exactly the same concentration. Thus, batch effects (i.e., disparities in electrode fabrication and dsDNA immobilization) are minimized due to the use of paired data in our assays.

By using the standard deviation of all electrodes incubated with mouse IgGs, we determined the limit of detection (LOD) of our sensor as the background signal (i.e., the average of mouse IgGincubated electrodes) plus three standard deviations. We obtained a LOD of $0.1 \mu \mathrm{g} / \mathrm{mL}$ of $\alpha$ dsDNA antibodies, corresponding to a measured current of - $0.1 \mu \mathrm{A}$. Currents (cathodic) higher than that could be taken into account to discriminate samples that contain $\alpha$-dsDNA antibodies.

\section{Performance in serum samples}

202 Once addressing the sensitivity and specificity of the sensor towards $\alpha$-dsDNA antibody detection 203 in buffer, we wondered whether this device would be able to detect these antibodies in a complex sample mixture such as serum. It is reported that many sensors fail to give a wellresponse signal when exposed to real complex matrixes even if their performance indicates the recognition of specific analytes when using pure laboratory samples. ${ }^{30}$ To test this, we 
207 performed standard additions of the monoclonal $\alpha$-dsDNA antibody (or irrelevant antibodies) in

$2081 / 80$ dilutions of fetal bovine serum. We decided to work in $1 / 80$ serum as this is the dilution

209 used to differentiate positive from negative samples in SLE ${ }^{14,15}$. Even though we could no

210 longer detect a clear trend in the electrical current as a function of the specific antibody

211 concentration, we did observe a statistically significant $(p<0.0001)$ higher reduction (i.e.,

212 negative) current of TMB in electrodes incubated with $\alpha$-dsDNA-containing FBS vs. mouse

213 IgG-containing FBS (Figure 4A). This also depended on the presence of immobilized dsDNA

214 on the surface of the electrodes, as electrodes modified with BSA alone showed background

215 currents even when incubated with $\alpha$-dsDNA-containing FBS (Figure 4A, diamonds).

216 Assuming a cutoff value of $0.1 \mu \mathrm{A}$ (which corresponds to the measured current at the LOD), the

217 biosensor was $100 \%$ specific (no false positives) and $82 \%$ sensitive (3 false negatives out of

218 17). Similar results were obtained for current measurements at $50 \mathrm{~s}$ and for quantization of the

219 electrical charge passed through the electrode during 100s (Supplementary Figure 3)

220 Our next attempt was to reduce assay time and manipulation steps, in order to ease

221 automatization of the sensor in the future. We hypothesized that by adding the conjugated ABs

222 directly into $\alpha$-dsDNA-containing FBS, we could then incubate both molecules above the

223 electrode surface in a single step, without the need for a washing step in the middle. This

224 method, called "one-step" in opposition to the "two-step" method, reduced the total assay time

225 to $30 \mathrm{~min}$ (vs. $60 \mathrm{~min}$ in the "two-step") without compromising specificity (Figure 4B), as

226 evident from a comparison of ROC curves for both methods (Figure 4C). In contrast, the

227 current separation between positive and negative samples was even higher, with a $100 \%$

228 specificity and $90 \%$ sensitivity when using the $0.1 \mu \mathrm{A}$ cutoff. To discard that the positives

229 results were not simply a consequence of using monoclonal antibodies instead of normal mouse

$230 \mathrm{IgG}$, we also analyzed other negative samples in which irrelevant monoclonal antibodies (e.g.,

231 anti-human CD9) were added to 1/80 FBS at the same concentration as the positive samples

232 (Figure 4B, triangles). Overall, these results show that our biosensor is capable of

233 discriminating between serum samples containing $\alpha$-dsDNA antibodies and those which do not. 
234 This is promising towards the future development of electrochemical biosensors for

235 autoimmune disease-based applications.

237 Discussion

238 There is a need for rapid, simple and low-cost analytical devices capable of determining the

239 presence and concentration of autoantibodies in blood specimens, to favor disease diagnosis,

240 treatment and monitoring. ${ }^{12,31-33}$ Currently, these assays are carried out in clinical laboratories

241 and demand time, costly equipment and specialized human resources. In contrast,

242 electrochemical biosensors are intrinsically compatible with point-of-care testing ${ }^{22,27,34}$, and that

243 makes them suitable for decentralized autoantibody determination. However, the number of

244 reports describing electrochemical biosensors for anti-dsDNA autoantibodies (a hallmark of

245 SLE and other autoimmune diseases) is rather scarce (Table 1).

246 Some of the important parameters to study when describing new analytical technologies or

247 methods are the sensitivity (often expressed as the limit of detection) and specificity of the

248 assays, as well as the performance of the method in real samples. Previous reports, such as the

249 seminal work performed by Rubin and Konstantinov, have used sandwich immunoassays in

250 fluidic devices to detect anti-dsDNA, anti-nuclear or anti-chromatin autoantibodies with

251 electrochemical biosensors based on a similar electrochemical readout as the used herein. ${ }^{23-25}$

252 The authors compared the amount of autoantibodies in patient serum samples, and stablished the

253 performance of their sensor against commercial ELISA kits. However, their output was based

254 on the value of the measured electrochemical current, so establishing comparisons of analytical

255 performances with other methods was difficult, if not impossible. To overcome this problem,

256 we performed calibration curves in artificial samples containing monoclonal anti-dsDNA

257 antibodies, as suggested by Buhl et al. (2009). ${ }^{2}$ We obtained a limit of detection [LOD] of 0.1

$258 \mu \mathrm{g}$ of $\alpha$-dsDNA antibodies per $\mathrm{mL}$, and used the current in the LOD to define a cut-off in non- 
259 human serum samples were known quantities of specific or irrelevant antibodies were spiked-in.

260 Through this procedure, we obtained a 90\% sensitivity and a 100\% specificity with the "onse-

261 step" method (i.e., we could label 9 out of 10 positive samples as positive, and 10 out of 10

262 negative samples as negative). Considering the fact that we used 1/80 dilutions of serum, our

263 real detection limit increases up to $8 \mu \mathrm{g} / \mathrm{mL}$, which is slightly lower but comparable to previous

264 reports $^{23}$

265 Since reported detection limits are usually based on the title of autoantibodies (i.e., 1 /

266 maximum dilution of a serum sample for which a positive signal is still detected) we wondered

267 whether our detection limit of $8 \mu \mathrm{g} / \mathrm{mL}$ (16 ng total anti-dsDNA antibody, since sample

268 volume is only $2 \mu \mathrm{L}$ ) was clinically relevant. To study this, we performed serial dilutions of

269 FBS spiked-in with known amounts of $\alpha$-dsDNA antibodies, and looked for the amount which

270 mimicked serial dilutions of a SLE patient serum by ELISA. By doing so, we estimated the $\alpha$ -

271 dsDNA cargo in this patient sample to be precisely $8.8 \mu \mathrm{g} / \mathrm{mL}$. Thus, the limit of detection of

272 our sensor is in principle compatible with clinical applications. Further optimization will be

273 needed to achieve this goal, as mammalian non-human antibodies and serum were used in this

274 study.

275 One of the important aspects of our study is the minimization of assay time and manipulation

276 steps achieved with the thus called "one-step" method. If electrochemical biosensors are chosen

277 for their compatibility with point-of-care tests, they should be compatible with automatization in

278 order to avoid the need of specialized technicians. By decreasing the number of manipulation

279 steps (including sample incubation, washes, addition of reactants, among others) we facilitate

280 the implementation of our sensing methodology in future analytical devices. By pre-incubating

281 serum samples with the HRP-conjugated secondary antibodies, we have successfully decreased

282 manipulation steps from 5 (sample incubation, wash, conjugate incubation, wash, TMB $/ \mathrm{H}_{2} \mathrm{O}_{2}$

283 addition and electrochemical measurement) to 3 (sample incubation, wash, addition of redox

284 mediator and electrochemical measurement). Furthermore, this protocol reduced total assay time

285 from 60 to 30 minutes. 


\section{Conclusion}

287 We provide a simple protocol for the electrochemical sensing of $\alpha$-dsDNA autoantibodies in 288 serum samples, with excellent distinction between samples containing or not $\alpha$-dsDNA 289 antibodies in concentrations comparable to those present in the sera of autoimmune disease 290 patients. The total assay time of 30 minutes and the few manipulation steps will aid in the 291 automatization of this protocol in order to obtain portable sensors for their use outside of 292 laboratory facilities.

293 Conflicts of Interest

\section{Acknowledgments}

296 The authors want to thank Eduardo Méndez (Faculty of Science, Uruguay) for helpful discussions and Alfonso Cayota (Institut Pasteur de Montevideo) for some of the antibodies used in this work. JPT is a member of the National System of Researchers (ANII, Uruguay) and the Program for the Development of Basic Science (PEDECIBA, Uruguay).

\section{References}

1 A. Lerner, P. Jeremias and T. Matthias, Int. J. Celiac Dis., 2016, 3, 151-155.

2 A. Buhl, S. Page, N. H. H. Heegaard, P. von Landenberg and P. B. Luppa, Biosens. Bioelectron., 2009, 25, 198-203.

3 K. N. Konstantinov, A. Tzamaloukas and R. L. Rubin, Autoimmun. Highlights, 2013, 4, $55-61$.

4 K. H. Costenbader, S. Gay, M. E. Alarcón-Riquelme, L. laccarino and A. Doria, Autoimmun. Rev., 2012, 11, 604-609.

5 E. Cozzani, M. Drosera, G. Gasparini, A. Parodi, E. Cozzani, M. Drosera, G. Gasparini and A. Parodi, Autoimmune Dis. Autoimmune Dis., 2014, 2014, 2014, e321359.

6 I. Fattal, N. Shental, D. Mevorach, J. M. Anaya, A. Livneh, P. Langevitz, G. ZandmanGoddard, R. Pauzner, M. Lerner, M. Blank, M. E. Hincapie, U. Gafter, Y. Naparstek, Y. 
Shoenfeld, E. Domany and I. R. Cohen, Immunology, 2010, 130, 337-343.

8 C. K. Li and D. A. Isenberg, Medicine (Baltimore)., 2006, 34, 445-452.

9 S. Ntali and M. Y. Karim, Transl. Res., 2010, 156, 317-319.

10 D. A. Isenberg, C. T. Ravirajan, A. Rahman and J. Kalsi, Lupus, 1997, 6, 290-304.

11 M. Petri, Rheum. Dis. Clin. North Am., 2005, 31, 245-254.

12 K. N. Konstantinov and R. L. Rubin, Autoimmun. Highlights, 2017, 8, 17-20.

13 F. Fiegel, A. Buhl, H.-P. Jaekel, E. Werle, M. Schmolke, M. Ollert and P. B. Luppa, Lupus, 2010, 19, 957-964.

14 O. G. Carballo, F. B. Ingénito, A. A. Ginaca, P. Carabajal, M. A. Costa and J. Balbaryski, 2012, 46, 3-13.

15 A. Dellavance, A. Gabriel Júnior, B. Nuccitelli, B. H. Taliberti, C. A. von Mühlen, C. D. A. Bichara, C. H. R. Dos Santos, C. Bueno, C. M. Yano, C. L. P. Mangueira, D. G. Carvalho, E. Cardoso, E. Bonfá, F. I. E. Araújo, G. G. Rassi, H. M. Mundim, I. Bendet, J. Rego, L. M. E. D. A. Vieira, L. E. C. Andrade, M. O. F. Barbosa, M. Sugiyama, M. B. Santiago, N. Slhessarenko, N. A. Da Silva, P. L. C. Francescantonio, R. Jarach, R. Suda, R. A. Levy, S. O. Sampaio, S. P. F. Neves, W. D. M. Cruvinel, W. S. Dos Santos and Y. K. D. M. Nóbrega, Rev. Bras. Reumatol., 2009, 49, 89-98.

16 N. J. Olsen, M. Y. Choi and M. J. Fritzler, Arthritis Res. Ther., 2017, 19, 172.

17 M. Mascini and S. Tombelli, Biomarkers, 2008, 13, 637-657.

18 G. S. Turner, A.P.F., Karube, I. and Wilson, Biosensors: Fundamentals and Applications., Oxford University Press, Oxford., 1987.

19 A. Buhl, J. H. Metzger, N. H. H. Heegaard, P. Von Landenberg, M. Fleck and P. B. Luppa, Clin. Chem., 2007, 53, 334-341.

20 N. M. do Nascimento, A. Juste-Dolz, E. Grau-García, J. A. Román-Ivorra, R. Puchades, A. Maquieira, S. Morais and D. Gimenez-Romero, Biosens. Bioelectron., 2017, 90, 166173.

21 R. F. Fakhrullin, V. G. Vinter, A. I. Zamaleeva, M. V. Matveeva, R. A. Kourbanov, B. K. Temesgen, D. G. Ishmuchametova, Z. I. Abramova, O. A. Konovalova and M. K. Salakhov, Anal. Bioanal. Chem., 2007, 388, 367-375.

22 A. P. F. Turner, Chem. Soc. Rev., 2013, 42, 3184.

23 R. L. Rubin, D. Wall and K. N. Konstantinov, Biosens. Bioelectron., 2014, 51, 177-183.

24 R. L. Rubin and K. N. Konstantinov, Biosens. Bioelectron., 2016, 83, 306-311.

25 K. N. Konstantinov, R. A. Sitdikov, G. P. Lopez, P. Atanassov and R. L. Rubin, Biosens. Bioelectron., 2009, 24, 1949-1954.

26 A. Debesa Padilla and O. Hernández Betancourt, Rev. Cuba. Investig. Biomédicas, 2012, 31, 467-479.

27 X. Doldán, P. Fagúndez, A. Cayota, J. Laíz and J. P. Tosar, Anal. Chem., 2016, 88, 10466-10473.

28 J. P. Tosar, J. L. Holmes, S. D. Collyer, F. Davis, J. Laíz and S. P. J. Higson, Biosens. Bioelectron., 2013, 41, 294-301. 
F. G. Cottrell, Z. Phys. Chem, 1903, 42, 385.

30 J. P. Tosar, G. Brañas and J. Laíz, Biosens. Bioelectron., 2010, 26, 1205-1217.

31 M. Sher, R. Zhuang, U. Demirci, W. Asghar, F. Atlantic, B. Raton, B. Raton, E. Detection, P. Alto and B. Raton, Expert Rev Mol Diagn, 2017, 17, 351-366.

32 M. Thaler, A. Buhl, H. Welter, A. Schreiegg, M. Kehrel, B. Alber, J. Metzger and P. B. Luppa, 2009, 1417-1429.

33 X. Zhang, A. Zambrano and Z. L. Yikun, Arch. Immunol. Ther. Exp. (Warsz)., 2017, 65, 111-121.

34 J. P. Tosar, K. Keel and J. Laíz, Biosens. Bioelectron., 2009, 24, 3036-3042.

35 Z. Li, F. Li, Y. Xing, Z. Liu, M. You, Y. Li, T. Wen, Z. Qu, X. Ling Li and F. Xu, Biosens. Bioelectron., 2017, 98, 478-485.

36 R. Nosrati, B. Golichenari, A. Nezami, S. M. Taghdisi, B. Karimi, M. Ramezani, K. Abnous and S. A. M. Shaegh, TrAC - Trends Anal. Chem., 2017, 97, 428-444.

37 L. A. Fraser, A. B. Kinghorn, R. M. Dirkzwager, S. Liang, Y. W. Cheung, B. Lim, S. C. C. Shiu, M. S. L. Tang, D. Andrew, J. Manitta, J. S. Richards and J. A. Tanner, Biosens. Bioelectron., 2018, 100, 591-596.

38 K. Papp, E. Holczer, C. Kecse-Nagy, Z. Szittner, V. Lóránd, P. Rovero, J. Prechl and P. Fürjes, Sensors Actuators, B Chem., 2017, 238, 1092-1097.

\section{Figure Legends}

Scheme 1. Detection strategy. Positive or negative serum samples (containing $1 / 80$ fetal bovine serum plus either murine monoclonal anti-dsDNA [red] or irrelevant [green] antibodies) were incubated with dsDNA-modified carbon electrodes, washed, and later incubated with antimouse IgG antibodies conjugated to HRP enzyme [blue] (A). In a more time-efficient and automatization-compatible strategy, conjugated antibodies were mixed with the serum samples before incubation (one-step, B).

Figure 1. Specificity of $\alpha$-dsDNA antibody by enzyme-linked immunosorbent assay. Plates were sensitized with vortexed (squares) or intact (circles) salmon sperm DNA and incubated with different concentrations of $\alpha$-dsDNA antibody (black) or mouse IgGs as a control (white).

\section{Figure 2. Electrode modification with dsDNA and electrochemical sensing of $\alpha$-dsDNA} antibodies. A) cyclic voltammograms showing guanine oxidation (arrow) in dsDNA-modified electrodes (solid line) vs buffer-treated (dashed line) screen-printed carbon electrodes. B) Intensity vs time curves for TMB reduction on the electrode surface at - $0.1 \mathrm{~V}$. dsDNAmodified BSA-blocked electrodes were incubated with $\alpha$-dsDNA antibodies at two different concentrations (solid lines), normal mouse IgG at $2.5 \mu \mathrm{g} / \mathrm{mL}$ (thick dashed line), or buffer (thin 
316 dashed line), and then incubated with $\alpha$-mouse IgG/HRP conjugates, followed by addition of a

$317 \mathrm{TMB} / \mathrm{H}_{2} \mathrm{O}_{2}$ solution.

318 Figure 3. Optical and electrochemical response of the sensor. A) Absorbance changes as a 319 function of the concentration of $\mathrm{m} \alpha$-dsDNA (black circles) or mouse IgGs (open circles). B)

320 Reduction current of TMB at 100s as function of the concentration of $\alpha$-dsDNA (black circles)

321 or mouse IgGs (open circles). LOD (limit of detection) was determined as the background

322 signal plus three standard deviations. For visualization purposes, the current axis direction was

323 inverted. Error bars correspond to the standard error of the mean of two independent replicates.

324 Bi-electrodes containing specific and irrelevant antibodies at the same concentration were

325 assayed in parallel.

326 Figure 4. "One-step" vs "two-step" method. Reduction current recorder at 100s after 327 incubation with serum samples containing either relevant or irrelevant antibodies at the same 328 concentration with the "two-step" (A) or "one-step" (B) method. To reduce batch effects, 329 specific (black circles) and irrelevant (mouse IgG: open circles; monoclonal $\alpha$-human CD9, 330 open triangles) antibody-containing samples were incubated in parallel in bi-electrode strips. 331 Diamonds correspond to carbon electrodes not modified with dsDNA (negative controls). Error 332 bars correspond to the standard error of the mean. Student $t$ test (two-tailed) was carried out to 333 test statistically significance in the difference between positives and negatives. The current 334 corresponding to the detection limit of the method was used as cut-off in order to establish assay 335 specificity and sensitivity. Alternatively, a mobile cut-off was used in order to obtain ROC 336 (receiving operating characteristic) curves for both methods $(\mathrm{C})$.

Table 1. Assay time and limit of detection of different biosensors for analysis of autoantibodies.

\begin{tabular}{|c|c|c|c|c|c|}
\hline \multirow{2}{*}{ Biosensor/Transductor } & Target & $\begin{array}{c}\text { Time assay } \\
(\mathbf{m i n})\end{array}$ & $\begin{array}{c}\text { Sample } \\
\text { amount* } \\
(\boldsymbol{\mu} \text { L) }\end{array}$ & LOD & Ref \\
\hline \multirow{2}{*}{ QCM } & $\alpha$-TRIM21(Ro52)/ $\alpha$-TROVE2(Ro60) & $>60$ & 1000 & $\mathrm{NR}$ & 20 \\
\cline { 2 - 6 } & $\alpha$-dsDNA & $\mathrm{NR}$ & 2000 & $\mathrm{NR}$ & 21 \\
\hline \multirow{2}{*}{ SPR } & $\alpha$-dsDNA & 15 & 45 & $\mathrm{NR}$ & $19 ; 13$ \\
\hline \multirow{3}{*}{ Electrochemical } & $\alpha$-chromatin & 30 & 200 & $\mathrm{NR}$ & 25 \\
\cline { 2 - 6 } & $\mathrm{ANNA}$ & 30 & 200 & $\mathrm{NR}$ & 24 \\
\cline { 2 - 6 } & $\alpha$-dsDNA & 30 & 200 & $10 \mu \mathrm{g} / \mathrm{mL}$ & 23 \\
\cline { 2 - 6 } & $\alpha$-dsDNA (two-step) & 60 & 2 & $8 \mu \mathrm{g} / \mathrm{mL}$ & This work \\
\hline
\end{tabular}


bioRxiv preprint doi: https://doi.org/10.1101/333641; this version posted May 30, 2018. The copyright holder for this preprint (which was not certified by peer review) is the author/funder, who has granted bioRxiv a license to display the preprint in perpetuity. It is made available under aCC-BY-ND 4.0 International license.

QCM: Quartz Crystal Microbalance. SPR: Surface Plasmon Resonance ANA: Total Antinuclear Antibody.

NR: not reported.

*Diluted serum samples 


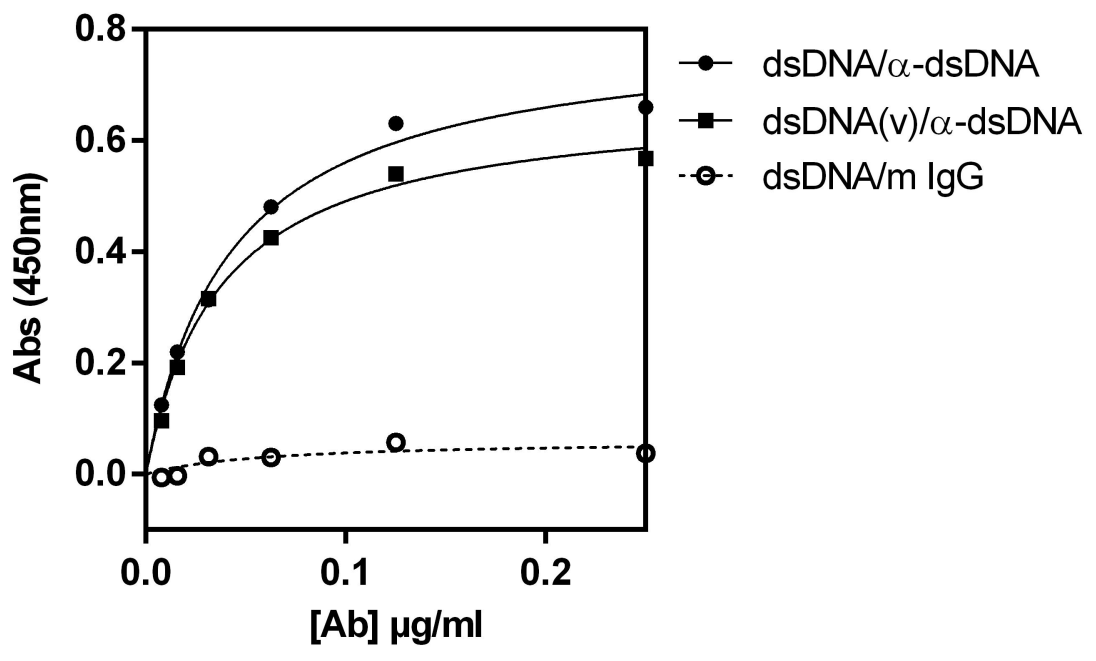


A

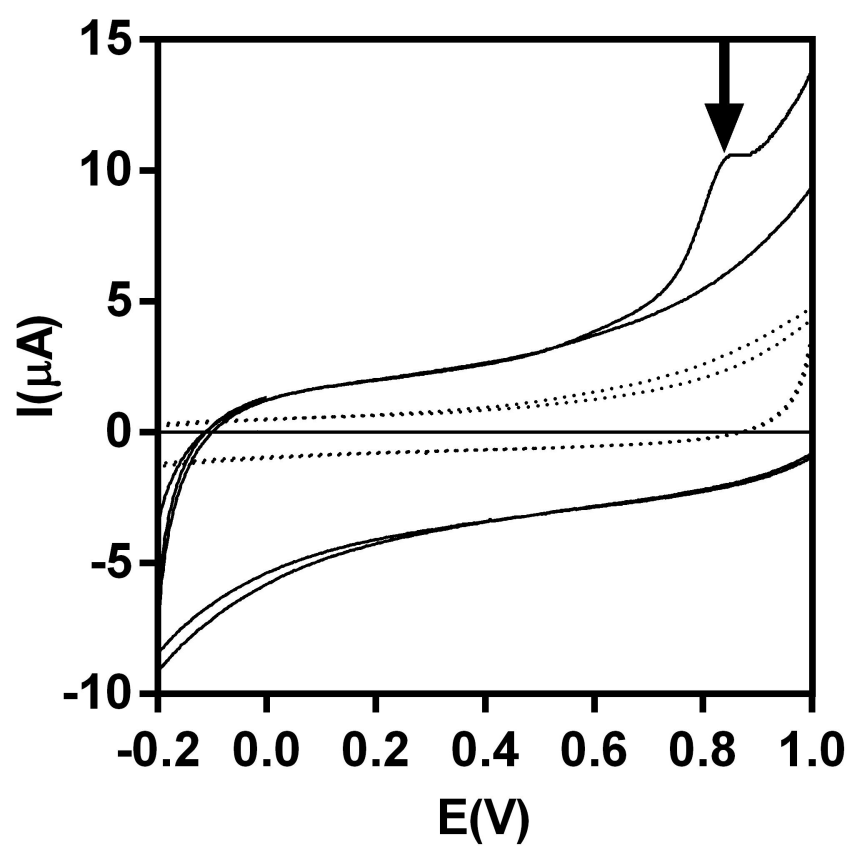

B

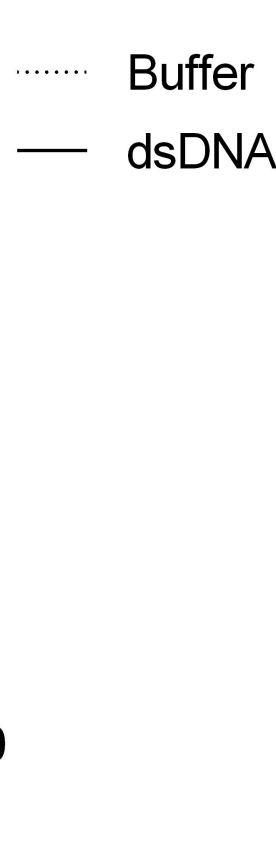

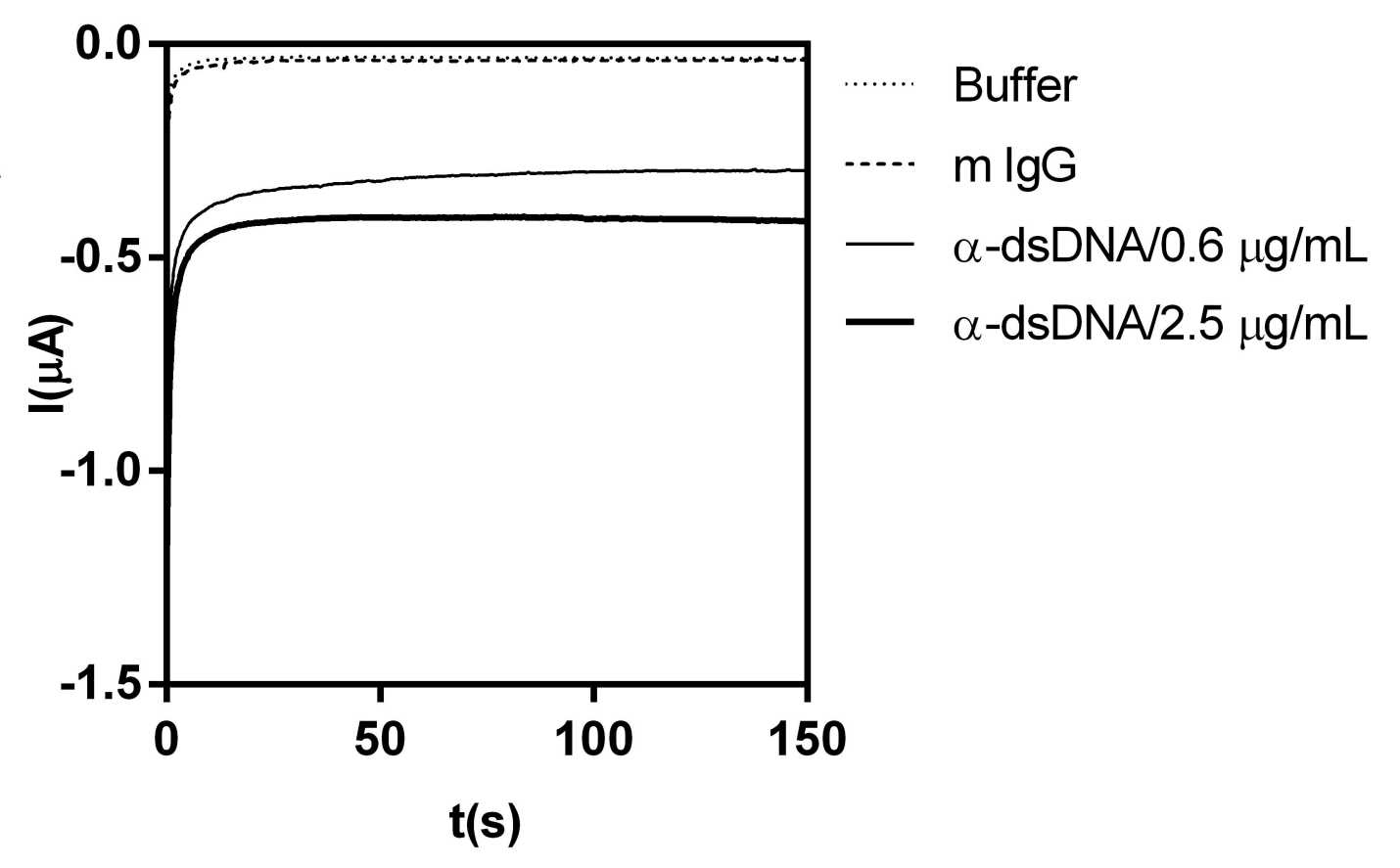


A

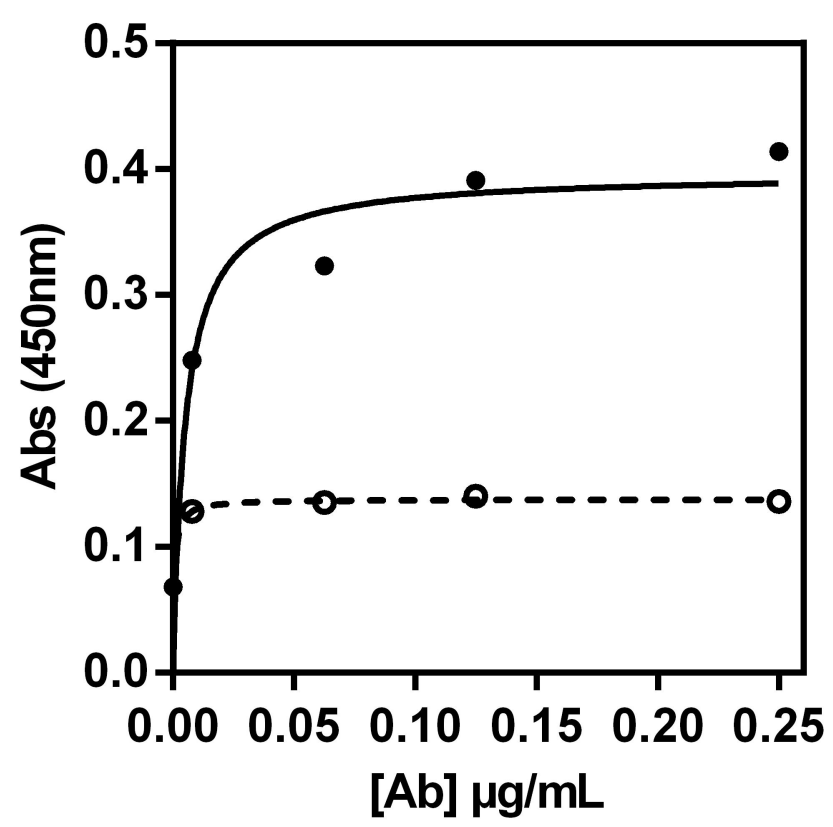

\section{B}

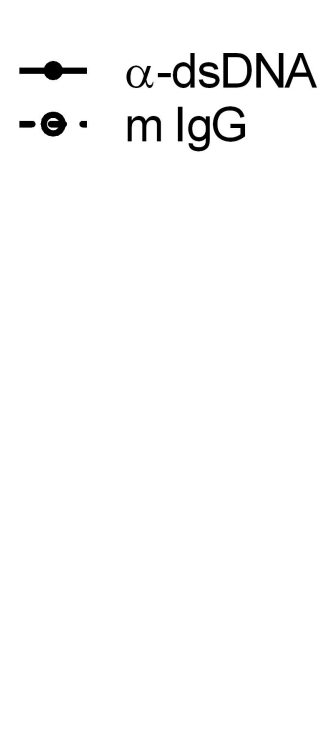

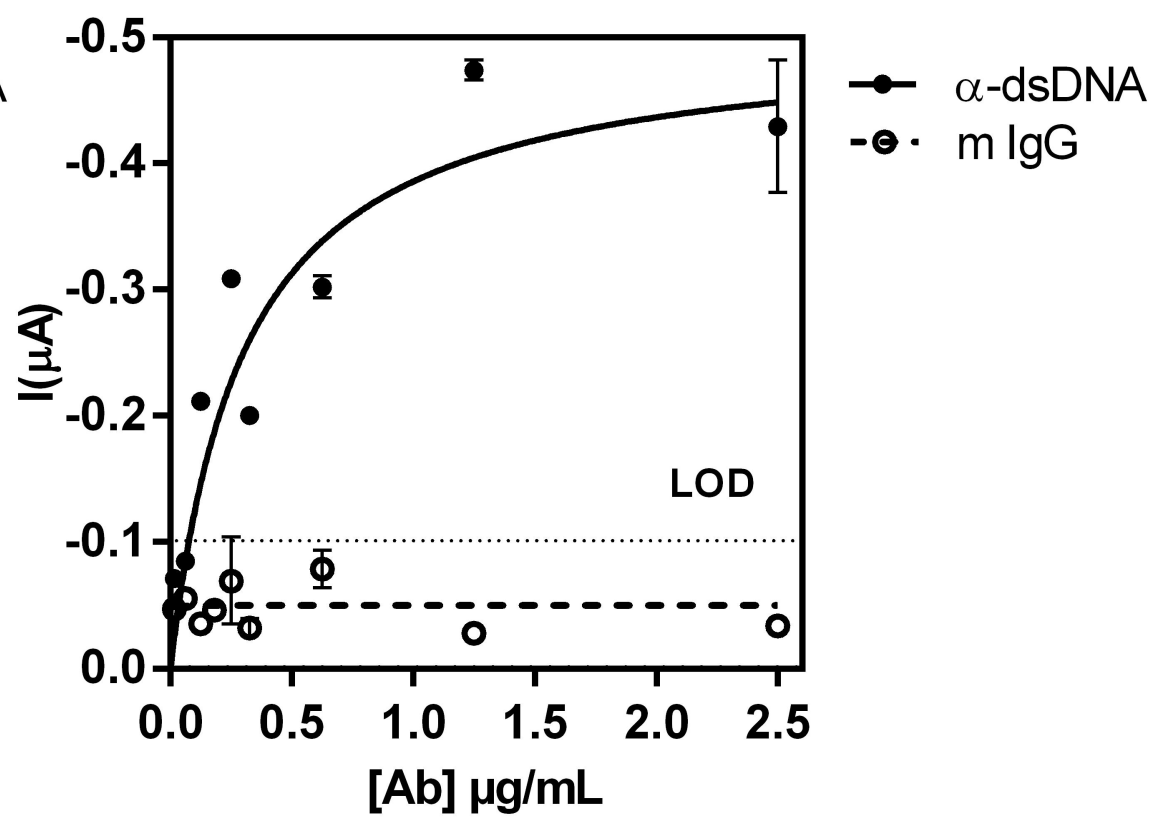


A

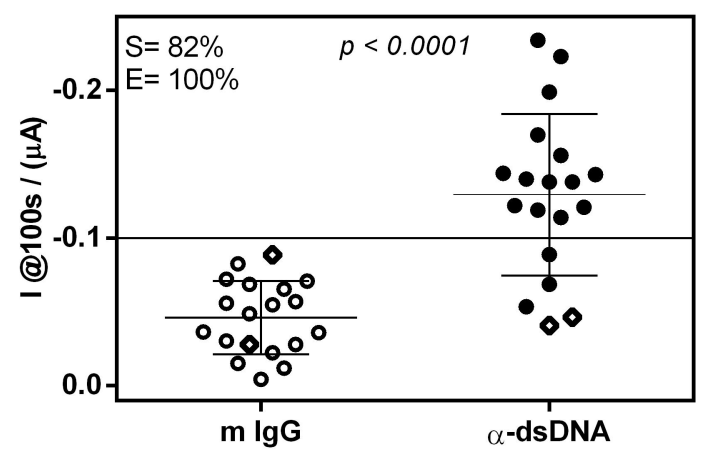

B

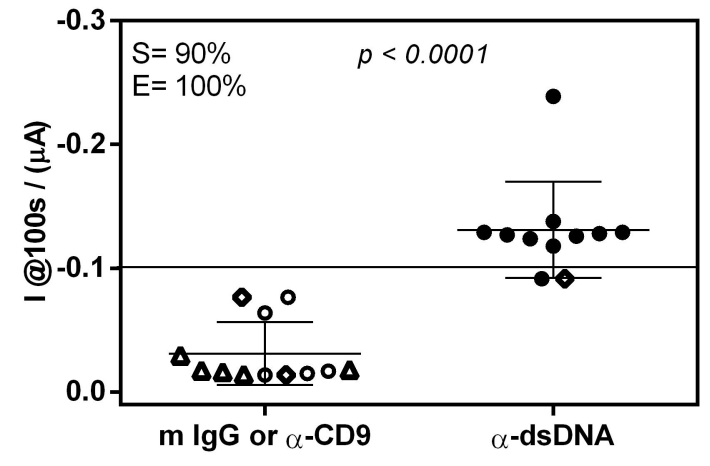

C

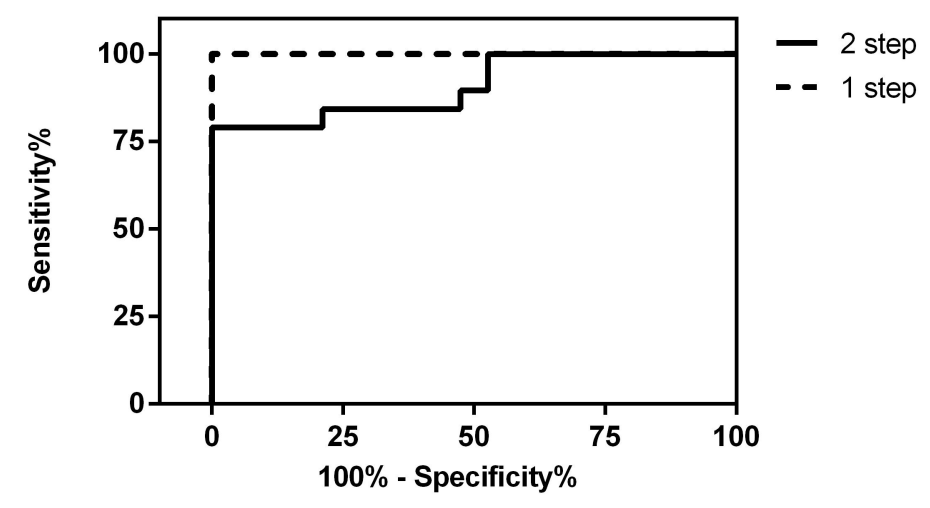


A: two-step method

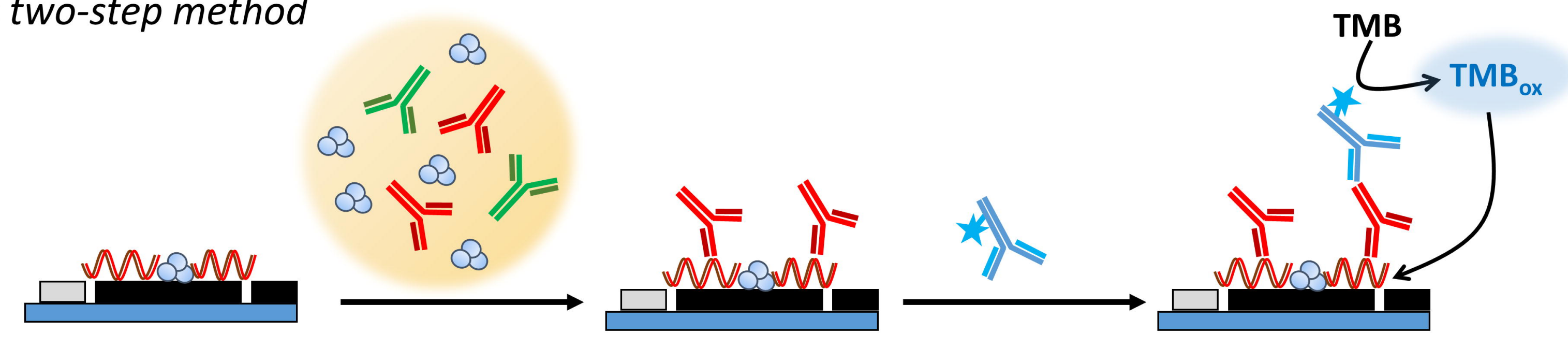

B: one-step method
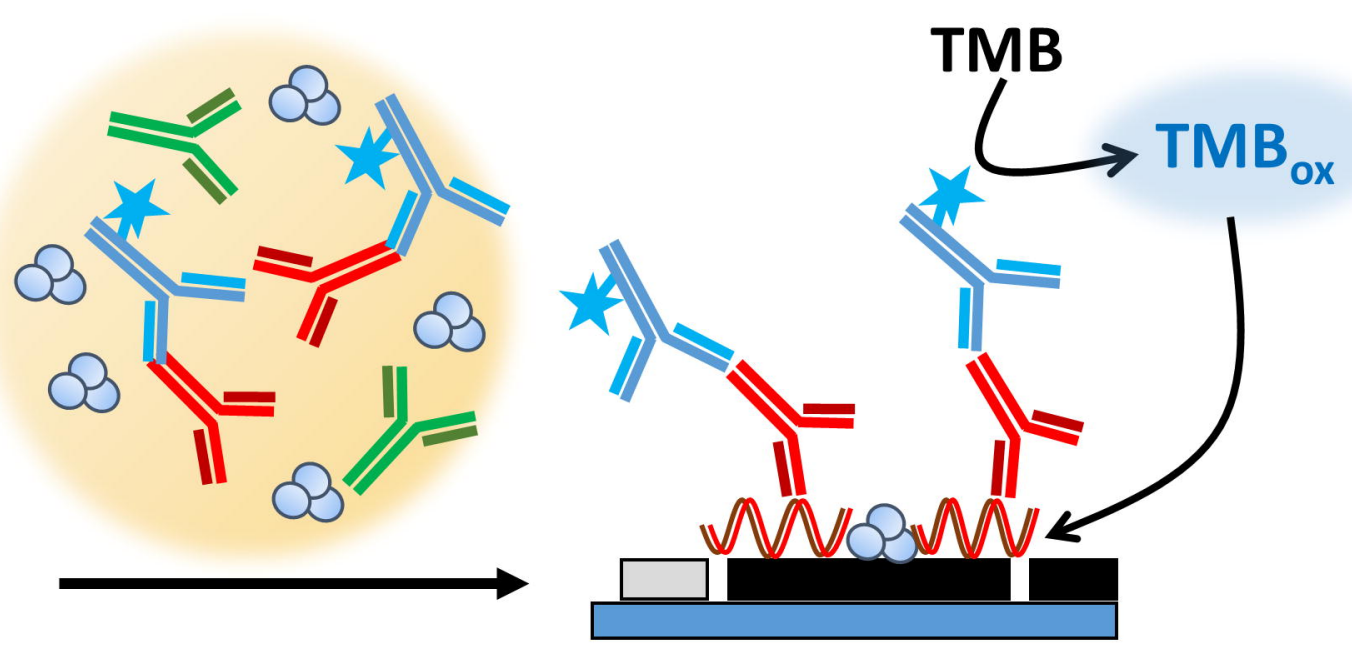

50\% REDUCTION:

- ASSAY TIME

- MANIPULATION STEPS 